\title{
Light (anti)nuclei production cross section studies in $p+C$ collisions at the NA61/SHINE experiment
}

\author{
Michał Naskręt ${ }^{a, *}$ on behalf of the NA61/SHINE Collaboration \\ (a complete list of authors can be found at the end of the proceedings) \\ ${ }^{a}$ University of Wroclaw \\ Wrocław, Poland \\ E-mail: michal.naskret@uwr.edu.pl
}

NA61/SHINE is a large-acceptance fixed-target experiment located at the CERN SPS, which studies final hadronic states in interactions of various particles and nuclei. It is unique in terms of providing data on a variety of collision systems at different collision energies. This allows for wide deuteron, antiproton, and antideuteron production cross-section studies. The latter is currently considered as a possible dark matter interaction signal with an exceptionally low background. The measurements of proton interactions with carbon target are important to reduce systematic experimental effects due to experiment-internal antideuteron production, as the most abundant element in the path of an incoming particle for the most detectors is carbon. This paper will focus on the analysis of NA61/SHINE data on $p+\mathrm{C}$ thin target collisions in the context of light (anti)nuclei production. I will present a performance study of analysis of experimental data, discuss quality cuts and the particle identification method.

$37^{\text {th }}$ International Cosmic Ray Conference (ICRC 2021)

July 12th-23rd, 2021

Online - Berlin, Germany

\footnotetext{
${ }^{*}$ Presenter
} 


\section{Introduction}

The NA61/SHINE physics program pursues many topics in different areas of physics. The core focus is on the Strong Interaction program with a scan of the collision system size and collision energy two-dimensional space. This is done in order to study the onset of deconfinement of strongly interacting matter and to search for the critical point of strongly interacting matter [1]. Additionally, cosmic ray and neutrino reference measurements are conducted. Hadron production measurements in $p+\mathrm{C}$ interactions were primarily performed in order to improve calculations of neutrino fluxes in T2K/J-PARC and Fermilab neutrino experiments as well as for simulations of cosmic-ray air showers in the Pierre Auger and KASCADE experiments.

The first results on $\pi^{ \pm}$and $K^{ \pm}$spectra produced in $p+\mathrm{C}$ interactions at $31 \mathrm{GeV} / c$ were already published by NA61/SHINE [2, 3] in 2011 and 2012. After an additional run in 2009, which took an eight times larger dataset, results on pion, kaon, and proton as well as $K_{\mathrm{S}}^{0}$ spectra in $p+\mathrm{C}$ interactions at $31 \mathrm{GeV} / c$ were published [4].

The latter data set can be used to support indirect dark matter signal searches. Investigating the flux of antiparticles in cosmic-rays is especially interesting because antiparticles do not have sizable astrophysical sources (see Fig. 1). An important source of background antideuterons for cosmic searches comes from interactions of $p$ and $\bar{p}$ with the interstellar medium [5]. $p+\mathrm{C}$ measurements are important to reduce systematic experimental effects due to experiment-internal antideuteron production, as the most abundant element in the path of an incoming particle for the AMS-02 experiment is carbon.

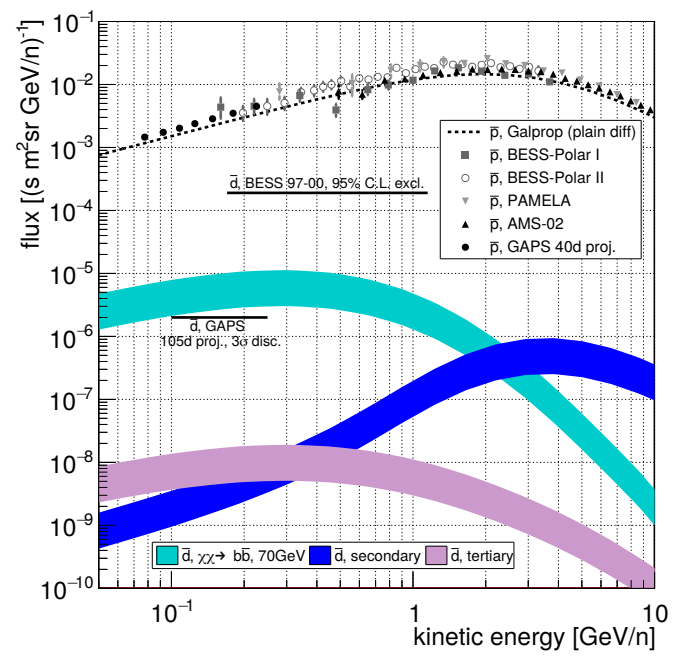

Figure 1: The predicted antideuteron flux corresponding to Dark Matter parameters indicated by AMS-02 antiproton signal as well as the predicted secondary and tertiary astrophysical antideuteron flux. Figure taken from Ref. [6].

\section{NA61/SHINE}

The NA61/SHINE detector (see Fig. 2) is a large-acceptance hadron spectrometer situated in the North Area H2 beam-line of the CERN SPS [7]. The main components of the detection 
system used in the analysis are four large volume Time Projection Chambers (TPC). Two of them, called Vertex TPCs (VTPC), are located downstream of the target inside superconducting magnets with a maximum combined bending power of $9 \mathrm{Tm}$. The main TPCs (MTPC) and two walls of pixel Time-of-Flight (ToF-L/R) detectors are placed symmetrically on either side of the beamline downstream of the magnets. The TPCs are filled with $\mathrm{Ar}: \mathrm{CO}_{2}$ gas mixtures in proportions 90:10 for the VTPCs and 95:5 for the MTPCs.

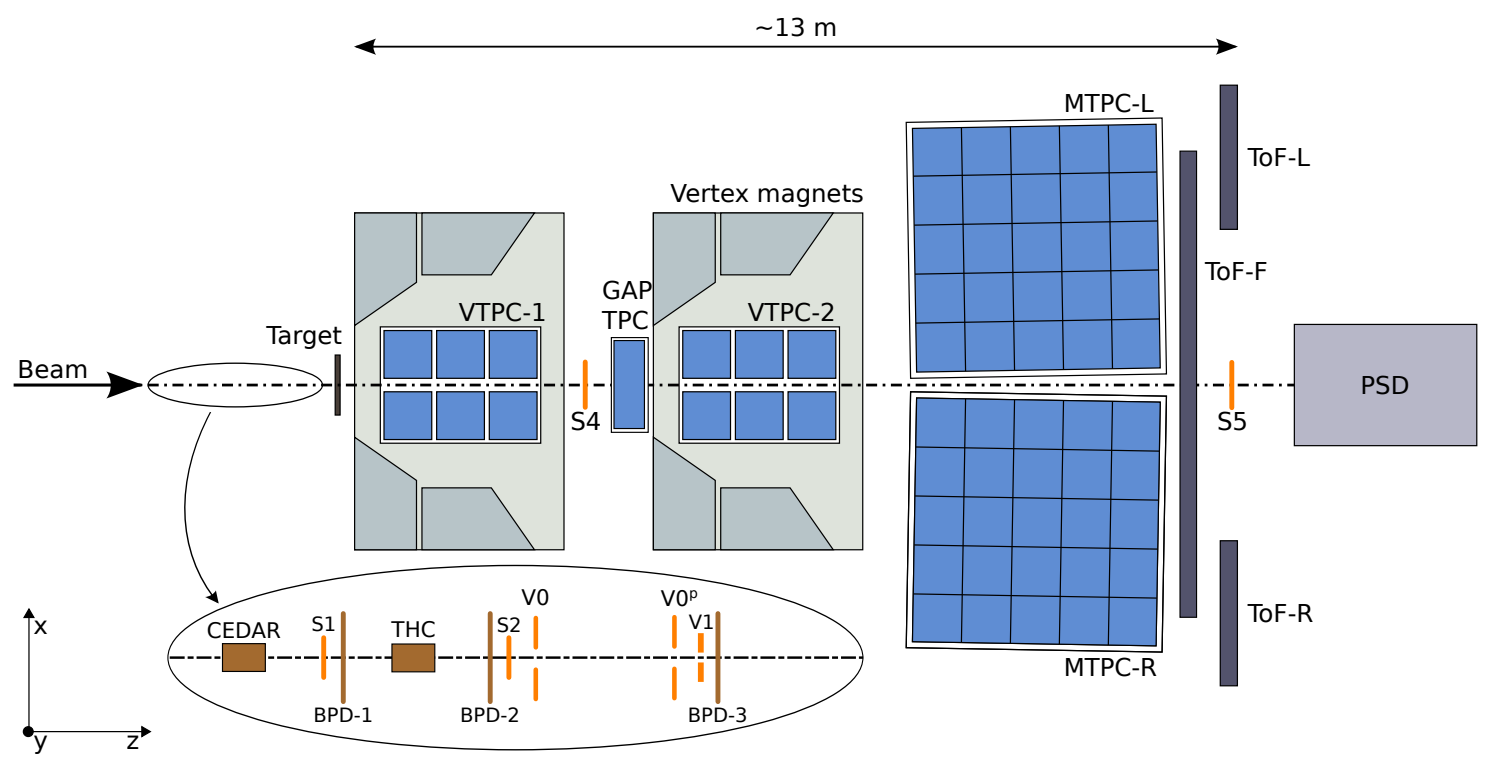

Figure 2: The schematic layout of the NA61/SHINE experiment at the CERN SPS [7] showing the components used for the $p+\mathrm{C}$ run (horizontal cut, not to scale). The trigger detector configuration upstream of the target is shown in the inset. Alignment of the chosen coordinate system is shown on the plot; its origin lies in the middle of VTPC-2, on the beam axis. The nominal beam direction is along the z-axis. The magnetic field bends charged particle trajectories in the $x-z$ (horizontal) plane. The drift direction in the TPCs is along the (vertical) y-axis.

\section{Analysis}

Reconstruction and calibration algorithms applied to the 2009 data are summarized in Ref. [2]. They resulted in good data quality suitable for the analysis (see e.g. Ref. [8]). The procedure was repeated recently for the $p+\mathrm{C}$ data set with the newest version of the reconstruction software and updated calibration parameters.

Measurements of the specific energy loss $\mathrm{d} E / \mathrm{d} x$ of charged particles by ionization in the TPC gas are used for their identification. The $\mathrm{d} E / \mathrm{d} x$ of a particle is calculated as the $50 \%$ truncated mean of the charges of the clusters (points) on the track traversing the TPCs. The calibrated $\mathrm{d} E / \mathrm{d} x$ distributions as a function of particle momentum for positively and negatively charged particles are presented in Fig. 3. The Bethe-Bloch parametrization of the mean energy loss, scaled to the experimental data, is shown by the curves for positrons (electrons), pions, kaons, (anti)protons, and (anti)deuterons. The typical achieved $\mathrm{d} E / \mathrm{d} x$ resolution is about $4 \%$. 

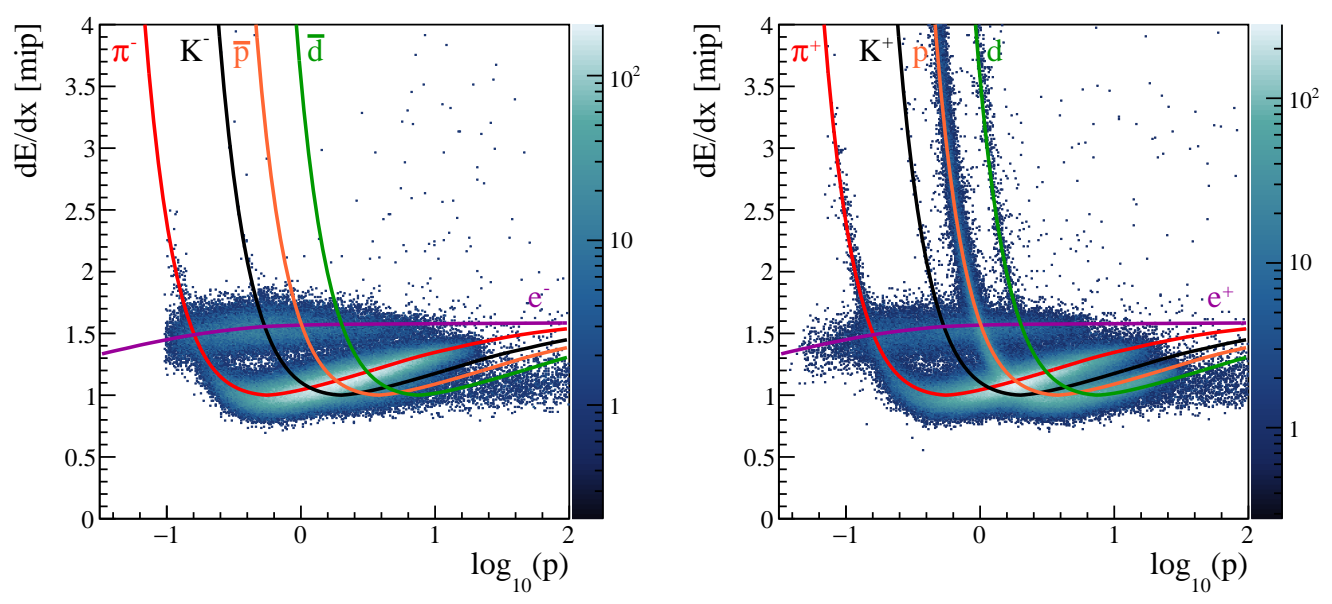

Figure 3: Specific energy loss $\mathrm{d} E / \mathrm{d} x$ in the TPCs for negatively (left) and positively (right) charged particles as a function of momentum. Curves show the Bethe-Bloch parameterizations of the mean $\mathrm{d} E / \mathrm{d} x$ calculated for different particle species. In the case of electrons and positrons, which reach the Fermi plateau, the mean $\mathrm{d} E / \mathrm{d} x$ is parameterized by a constant.

\subsection{Event and track cuts}

The dataset consists of $5.4 \cdot 10^{6}$ event triggers. To improve the rejection of out-of-target events the following event cuts were applied:

1. Requirement to have both the $\mathrm{x}$ and $\mathrm{y}$ positions of the beam particle measured by all three BPDs (Beam Position Detectors).

2. Rejection of events in which one or more additional beam particles are detected in the time window $t=[-2,0] \mu$ s before the triggering beam particle.

To ensure high reconstruction efficiency the following cuts were applied to tracks:

1. Track momentum fit at the interaction vertex should have converged,

2. The total number of reconstructed points on the track should be greater than 30 ,

3. At least 12 reconstructed points in the three TPCs that were used for momentum measurement (VTPC-1, VTPC-2 and GTPC),

4. Distance of closest approach of the fitted track to the interaction point (impact parameter) smaller than $4 \mathrm{~cm}(4 \mathrm{~cm})$ in the horizontal (vertical) plane,

5. The ratio of the number of fitted to potential clusters should be between 0.8 and 1.1.

\subsection{Particle identification}

In this analysis, a simplified approach to identify particles is used. Namely, a $2 \sigma$ wide band around the Bethe-Bloch curves will serve as the classification border together with a cut-off for low $\mathrm{d} E / \mathrm{d} x$ particles. The latter is used to distinguish the particles of interest from electrons and 
positrons. All particles within the band will be identified as the corresponding particle type. For the antideuterons and antiprotons, which suffer from low statistics, the same band as deuterons and protons, respectively, are used.

\subsection{Eliminating contribution of secondary particles}

To establish the contribution of deuterons produced in secondary processes, a specific analysis procedure has been developed. The contributions of particles produced in events with the main vertex $z$ coordinate before and after the target center are compared. The probability of deuteron production in the coalescence of primary particles is uniform along the target length. The additional contributions from the monotonously growing probability of secondary processes should be excluded. An overview of the idea is presented in Fig. 4 (left) and in form of an equation in Eq. 1.

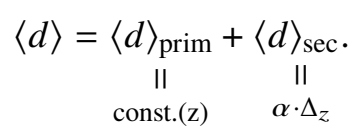
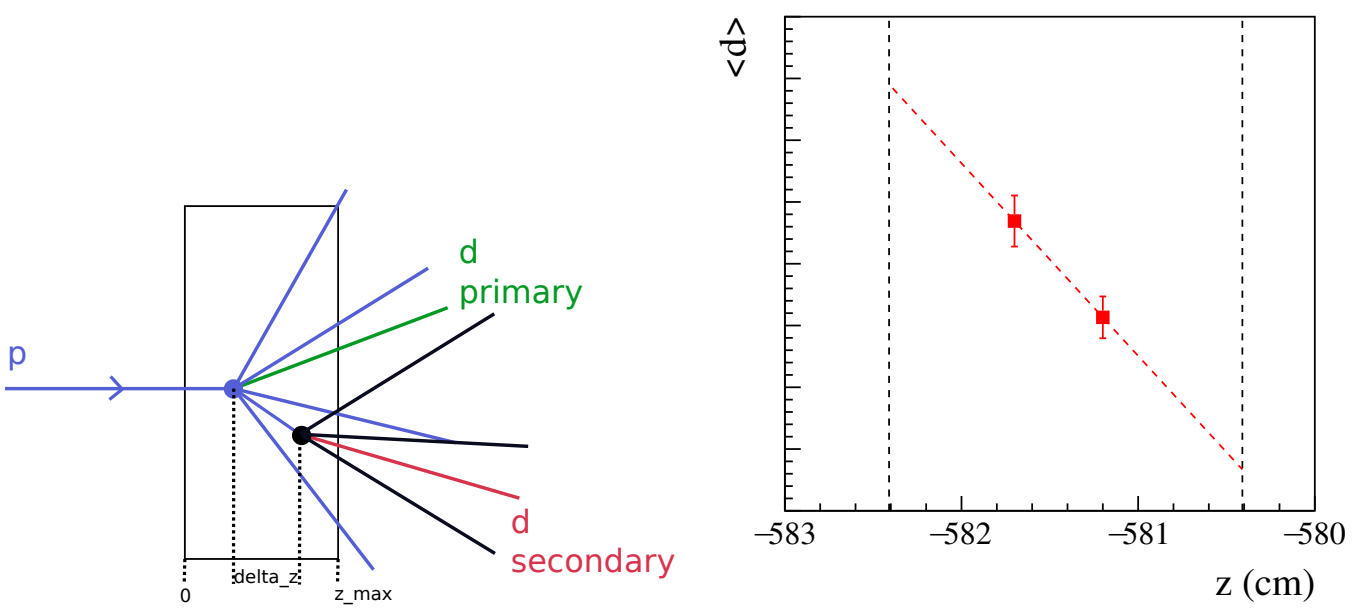

Figure 4: Left: An example of a possible $p+C$ interaction. The beam proton hits target atom and produces particles, among which are protons and deutrons - in this case called primary. A primary proton hits another target atom and produces next particles, among which is a deuteron - in this case called secondary. The latter is an unwanted contribution. Right: The mean number of deuterons $\langle d\rangle$ produced before and after target center (red boxes) with statistical uncertainties. The red dashed line is a connection of the points and black vertical dashed lines represent the boundaries of the carbon target.

To compensate for differences in detector acceptance using events with the main vertex $z$ coordinate before and after the target center, an acceptance map was used. $5 \mathrm{M}$ deuterons in flat phase-space $\left(y, p_{\mathrm{T}}\right.$ and $\left.\phi\right)$ were simulated and reconstructed to determine the detector efficiencies for the two cases. Next, only bins common for the two versions - before and after target center, were used. The common acceptance map was used to obtain $\langle d\rangle$.

Combining mean deuteron multiplicities $\langle d\rangle$ produced before and after target center with simulated z position of the true event vertex, it is possible to plot different contributions to Eq. 1. This is presented in Fig. 4 (right) and allows to extract the contribution $\langle d\rangle_{\text {prim }}$ which is of interest. 


\section{The coalescence model}

(Anti)deuteron production can be described within the coalescence model [9], which states that any (anti)proton and (anti)neutron within the sphere of radius $p_{0}$ in momentum space will coalesce to produce an (anti)deuteron. This is described by the following formula:

$$
\gamma_{d} \frac{\mathrm{d}^{3} N_{d}}{\mathrm{~d} p_{d}^{3}}=\frac{4 \pi}{3} p_{0}^{3}\left(\gamma_{p} \frac{\mathrm{d}^{3} N_{p}}{\mathrm{~d} p_{p}^{3}}\right)\left(\gamma_{n} \frac{\mathrm{d}^{3} N_{n}}{\mathrm{~d} p_{n}^{3}}\right)
$$

where $p_{i}$ and $\mathrm{d} N_{i} / \mathrm{d} p_{i}$ are, respectively, the momentum and the differential yield per event of particle $i$ ( $d=$ (anti)deuteron, $p=$ (anti)proton, $n=$ (anti)neutron) and the momentum space radius called the coalescence momentum $p_{0}$ is a phenomenological quantity, which cannot be calculated from first principles. However, $p_{0}$ does not only describe the required difference in momenta of the coalescence partners, but in reality parametrizes a number of other effects and conditions as well.

In order to simulate deuteron production in a collision, a coalescence afterburner was introduced and applied for events generated with Epos1.99 model (version CRMC 1.5.3) [10-12]. Two particles coalesce if the following condition is met in the center of mass frame of reference:

$$
\left|\vec{k}_{1}-\vec{k}_{2}\right|<2 p_{0}
$$

where

$$
p_{0}=\frac{A}{1+\exp B-\ln (T / C)}
$$

with $T$ being collision energy in $\mathrm{GeV}, A=89.6, B=6.6$ and $C=0.73$ for deuterons as described in Ref. [13].

The simulation allows for generating a correction factor for the detector acceptance due to geometry and reconstruction effects. The multiplicative correction factor is calculated as the ratio of the mean number of generated deuterons to the mean number of selected deuterons:

$$
c=\frac{\langle d\rangle_{\mathrm{gen}}}{\langle d\rangle_{\mathrm{sel}, \text { common acc }}} .
$$

The correction is applied in the following way:

$$
\langle d\rangle_{\text {final }}=c \cdot\langle d\rangle_{\text {pritm, common acc }}
$$

\section{Calculating the cross-section}

NA61/SHINE has already calculated and published the $p+C$ interaction trigger probability and resulting "trigger" cross-section value:

$$
\sigma_{\text {trig }}=305.7 \pm 2.7 \text { (stat) } \pm 1.0(\text { det }) \mathrm{mb},
$$

with (stat) being statistical and (det) systematic uncertainty. Using the latter it is possible to calculate the deuteron production cross-section with its statistical uncertainty from the following formula:

$$
\sigma_{d}=\frac{\sigma_{\text {trig }}}{f_{\text {prod }}(1-\epsilon)}\left(\frac{n_{d}^{I}}{N_{\text {trig }}^{\mathrm{I}}}-\epsilon \frac{n_{d}^{R}}{N_{\text {trig }}^{\mathrm{R}}}\right)
$$


where $N^{\mathrm{I}}$ and $N^{\mathrm{R}}$ are the numbers of events with the target inserted and removed, respectively, $n_{d}^{\mathrm{I}}$ and $n_{d}^{\mathrm{R}}$ number of deuterons produced with target inserted and removed, respectively. $\epsilon=0.123 \pm 0.004$ is the ratio of the interaction probabilities for operation with the target removed and inserted, and $f_{\text {prod }}=0.993$ is the fraction of production events.

Applying this method allows calculating the deuteron production cross-section in $p+\mathrm{C}$ collisions, but further analysis is still necessary.

Applying similar methods in order to calculate yields of $\bar{p}$ and $\bar{d}$ in the case of the $p+\mathrm{C}$ dataset does not give a satisfactory number of entries. This causes statistical uncertainties to be significantly above $10 \%$ and prevents meaningful inference. In the case of $\bar{p}$, the total multiplicity obtained with the simplified $d E / d x$ method was 27 , which results in a statistical uncertainty of about $20 \%$. In the case of $\bar{d}$ there were 9 candidates, resulting in a statistical uncertainty of $\sim 30 \%$. In order to reduce the statistical uncertainties below $10 \%$ the amount of data would have to be increased fourfold in case of $\bar{p}$ and twelve-fold in case of $\bar{d}$.

\section{Conclusions}

This work presents a performance study of the deuteron production cross-section measurements in $p+\mathrm{C}$ collisions with the NA61/SHINE experiment. An overview of cuts on events and tracks as well as a description of the simplified $d E / d x$ method was given. Details of the coalescence model together with a description of a Monte-Carlo-based correction for detector geometry were presented. Using this method it will only be possible to obtain results on $d$ yields and production cross-section. For $\bar{p}$ and $\bar{d}$, the event statistics were too low and would have to be significantly increased in order to obtain meaningful results. This might become possible in further data-taking campaigns. NA61/SHINE provides a source of valuable information on many collision systems, which may be utilized for reference measurements in indirect dark matter searches.

\section{Acknowledgments}

This work was supported by the Polish National Science Centre grant 2017/27/N/ST2/01009.

\section{References}

[1] N. Antoniou et al. Study of hadron production in hadron nucleus and nucleus nucleus collisions at the CERN SPS, 2006. CERN-SPSC-2006-034.

[2] N. Abgrall et al. Measurements of Cross Sections and Charged Pion Spectra in Proton-Carbon Interactions at $31 \mathrm{GeV} / c$. Phys. Rev., C84:034604, 2011.

[3] N. Abgrall et al. Measurement of Production Properties of Positively Charged Kaons in Proton-Carbon Interactions at 31 GeV/c. Phys. Rev., C85:035210, 2012.

[4] N. Abgrall et al. Measurements of $\pi^{ \pm}, K^{ \pm}, K_{S}^{0}, \Lambda$ and proton production in proton-carbon interactions at $31 \mathrm{GeV} / \mathrm{c}$ with the NA61/SHINE spectrometer at the CERN SPS. Eur. Phys. J. C, 76(2):84, 2016. 
[5] R. Duperray et al. Flux of light antimatter nuclei near Earth, induced by cosmic rays in the Galaxy and in the atmosphere. Phys. Rev., D71:083013, 2005.

[6] P. von Doetinchem et al. Cosmic-ray antinuclei as messengers of new physics: status and outlook for the new decade. JCAP, 08:035, 2020.

[7] N. Abgrall et al. NA61/SHINE facility at the CERN SPS: beams and detector system. JINST, 9:P06005, 2014.

[8] N. Abgrall et al. Measurement of negatively charged pion spectra in inelastic $\mathrm{p}+\mathrm{p}$ interactions at $p_{\text {lab }}=20,31,40,80$ and $158 \mathrm{GeV} / \mathrm{c}$. Eur.Phys.J., C74:2794, 2014.

[9] A. Schwarzschild et al. Production of tritons, deuterons, nucleons, and mesons by 30-gev protons on A1, Be, and Fe targets. Phys. Rev., 129:854-862, Jan 1963.

[10] Klaus Werner, Fu-Ming Liu, and Tanguy Pierog. Parton ladder splitting and the rapidity dependence of transverse momentum spectra in deuteron-gold collisions at RHIC. Phys. Rev., C74:044902, 2006.

[11] T. Pierog and K. Werner. EPOS Model and Ultra High Energy Cosmic Rays. Nucl.Phys.Proc.Suppl., 196:102-105, 2009.

[12] T. Pierog, 2018. private communication.

[13] Anirvan Shukla, Amaresh Datta, Philip von Doetinchem, Diego-Mauricio Gomez-Coral, and Carina Kanitz. Large-scale Simulations of Antihelium Production in Cosmic-ray Interactions. Phys. Rev. D, 102(6):063004, 2020. 


\section{Full Authors List: NA61/SHINE Collaboration}

A. Acharya ${ }^{9}$, H. Adhikary ${ }^{9}$, K.K. Allison ${ }^{25}$, E.V. Andronov ${ }^{21}$, T. Antićić ${ }^{3}$, V. Babkin ${ }^{19}$, M. Baszczyk ${ }^{13}$, S. Bhosale ${ }^{10}$, A. Blondel ${ }^{4}$, M. Bogomilov ${ }^{2}$, A. Brandin ${ }^{20}$, A. Bravar ${ }^{23}$, W. Bryliński ${ }^{17}$, J. Brzychczyk ${ }^{12}$, M. Buryakov ${ }^{19}$, O. Busygina ${ }^{18}$, A. Bzdak ${ }^{13}$, H. Cherif ${ }^{6}$, M. Ćirković $^{22}$, M. Csanad ${ }^{7}$, J. Cybowska ${ }^{17}$, T. Czopowicz ${ }^{9,17}$, A. Damyanova ${ }^{23}$, N. Davis ${ }^{10}$, M. Deliyergiyev ${ }^{9}$, M. Deveaux ${ }^{6}$, A. Dmitriev ${ }^{19}$, W. Dominik ${ }^{15}$, P. Dorosz ${ }^{13}$, J. Dumarchez ${ }^{4}$, R. Engel $^{5}$, G.A. Feofilov ${ }^{21}$, L. Fields ${ }^{24}$, Z. Fodor ${ }^{7,16}$, A. Garibov ${ }^{1}$, M. Gaździcki ${ }^{6,9}$, O. Golosov $^{20}$, V. Golovatyuk ${ }^{19}$, M. Golubeva ${ }^{18}$, K. Grebieszkow $^{17}$, F. Guber ${ }^{18}$, A. Haesler ${ }^{23}$, S.N. Igolkin ${ }^{21}$, S. Ilieva ${ }^{2}$, A. Ivashkin ${ }^{18}$, S.R. Johnson ${ }^{25}$, K. Kadija ${ }^{3}$, N. Kargin ${ }^{20}$, E. Kashirin ${ }^{20}$, M. Kiełbowicz ${ }^{10}$, V.A. Kireyeu ${ }^{19}$, V. Klochkov ${ }^{6}$, V.I. Kolesnikov ${ }^{19}$, D. Kolev ${ }^{2}$, A. Korzenev ${ }^{23}$, V.N. Kovalenko ${ }^{21}$, S. Kowalski ${ }^{14}$, M. Koziel ${ }^{6}$, B. Kozłowski ${ }^{17}$, A. Krasnoperov ${ }^{19}$, W. Kucewicz ${ }^{13}$, M. Kuich ${ }^{15}$, A. Kurepin ${ }^{18}$, D. Larsen ${ }^{12}$, A. László ${ }^{7}$, T.V. Lazareva ${ }^{21}$, M. Lewicki ${ }^{16}$, K. Lojek ${ }^{12}$, V.V. Lyubushkin ${ }^{19}$, M. Maćkowiak-Pawłowska ${ }^{17}$,Z. Majka ${ }^{12}$, B. Maksiak ${ }^{11}$, A.I. Malakhov ${ }^{19}$, A. Marcinek ${ }^{10}$, A.D. Marino ${ }^{25}$, K. Marton ${ }^{7}$, H.-J. Mathes ${ }^{5}$, T. Matulewicz ${ }^{15}$, V. Matveev ${ }^{19}$, G.L. Melkumov ${ }^{19}$, A.O. Merzlaya ${ }^{12}$, B. Messerly ${ }^{26}$, Ł. Mik ${ }^{13}$, S. Morozov ${ }^{18,20}$, Y. Nagai ${ }^{25}$, M. Naskręt ${ }^{16}$, V. Ozvenchuk ${ }^{10}$, V. Paolone ${ }^{26}$, O. Petukhov ${ }^{18}$, I. Pidhurskyi ${ }^{6}$, R. Płaneta ${ }^{12}$, P. Podlaski ${ }^{15}$, B.A. Popov ${ }^{19,4}$, B. Porfy ${ }^{7}$, M. Posiadała-Zezula ${ }^{15}$, D.S. Prokhorova ${ }^{21}$, D. Pszczel ${ }^{11}$, S. Puławski ${ }^{14}$, J. Puzović ${ }^{22}$, M. Ravonel ${ }^{23}$, R. Renfordt ${ }^{6}$, D. Röhrich ${ }^{8}$, E. Rondio ${ }^{11}$, M. Roth ${ }^{5}$, B.T. Rumberger ${ }^{25}$, M. Rumyantsev ${ }^{19}$, A. Rustamov ${ }^{1,6}$, M. Rybczynski ${ }^{9}$, A. Rybicki ${ }^{10}$, S. Sadhu ${ }^{9}$, A. Sadovsky ${ }^{18}$, K. Schmidt ${ }^{14}$, I. Selyuzhenkov ${ }^{20}$, A.Yu. Seryakov ${ }^{21}$, P. Seyboth ${ }^{9}$, M. Słodkowski ${ }^{17}$, P. Staszel ${ }^{12}$, G. Stefanek ${ }^{9}$, J. Stepaniak ${ }^{11}$, M. Strikhanov ${ }^{20}$, H. Ströbele ${ }^{6}$, T. Šuša ${ }^{3}$, A. Taranenko ${ }^{20}$, A. Tefelska ${ }^{17}$, D. Tefelski ${ }^{17}$, V. Tereshchenko ${ }^{19}$, A. Toia ${ }^{6}$, R. Tsenov ${ }^{2}$, L. Turko $^{16}$, R. Ulrich ${ }^{5}$, M. Unger ${ }^{5}$, D. Uzhva ${ }^{21}$, F.F. Valiev ${ }^{21}$, D. Veberič ${ }^{5}$, V.V. Vechernin ${ }^{21}$, A. Wickremasinghe ${ }^{26,24}$, K. Wójcik ${ }^{14}$, O. Wyszyński ${ }^{9}$, A. Zaitsev ${ }^{19}$, E.D. Zimmerman ${ }^{25}$, and R. Zwaska ${ }^{24}$

${ }^{1}$ National Nuclear Research Center, Baku, Azerbaijan

${ }^{2}$ Faculty of Physics, University of Sofia, Sofia, Bulgaria

${ }^{3}$ Ruđer Bošković Institute, Zagreb, Croatia

${ }^{4}$ LPNHE, University of Paris VI and VII, Paris, France

${ }^{5}$ Karlsruhe Institute of Technology, Karlsruhe, Germany

${ }^{6}$ University of Frankfurt, Frankfurt, Germany

${ }^{7}$ Wigner Research Centre for Physics of the Hungarian Academy of Sciences, Budapest, Hungary

${ }^{8}$ University of Bergen, Bergen, Norway

${ }^{9}$ Jan Kochanowski University in Kielce, Poland

${ }^{10}$ Institute of Nuclear Physics, Polish Academy of Sciences, Cracow, Poland

${ }^{11}$ National Centre for Nuclear Research, Warsaw, Poland

12 Jagiellonian University, Cracow, Poland

${ }^{13}$ AGH - University of Science and Technology, Cracow, Poland

${ }^{14}$ University of Silesia, Katowice, Poland

${ }^{15}$ University of Warsaw, Warsaw, Poland

${ }^{16}$ University of Wrocław, Wrocław, Poland

${ }^{17}$ Warsaw University of Technology, Warsaw, Poland

${ }^{18}$ Institute for Nuclear Research, Moscow, Russia

${ }^{19}$ Joint Institute for Nuclear Research, Dubna, Russia 
${ }^{20}$ National Research Nuclear University (Moscow Engineering Physics Institute), Moscow, Russia

${ }^{21}$ St. Petersburg State University, St. Petersburg, Russia

${ }^{22}$ University of Belgrade, Belgrade, Serbia

${ }^{23}$ University of Geneva, Geneva, Switzerland

${ }^{24}$ Fermilab, Batavia, USA

${ }^{25}$ University of Colorado, Boulder, USA

${ }^{26}$ University of Pittsburgh, Pittsburgh, USA 